\title{
A comparison of $n s$ and ps steam laser cleaning of Si surfaces
}

\author{
M. Mosbacher ${ }^{1, *}$, N. Chaoui ${ }^{2}$, J. Siegel ${ }^{2}$, V. Dobler ${ }^{1}$, J. Solis ${ }^{2}$, J. Boneberg ${ }^{1}$, C.N. Afonso ${ }^{2}$, P. Leiderer ${ }^{1}$ \\ ${ }^{1}$ University of Konstanz, Faculty of Physics, Fach M 676, 78457 Konstanz, Germany \\ ${ }^{2}$ Instituto de Optica, CSIC, Serrano 121, 28006 Madrid, Spain
}

Received: 21 July 1999/Accepted: 5 Oktober 1999/Published online: 22 December 1999

\begin{abstract}
We report a quantitative investigation on the efficiency of the steam laser cleaning process using ns and ps pulses. Well-characterized polymer particles with a diameter of $800 \mathrm{~nm}$ dispersed on commercial Si wafers were chosen as a modeling contaminant system. As a result of our investigation, we show for the first time the feasibility of performing efficient steam laser cleaning with ps laser pulses and compare the achieved efficiency with the one obtained for ns pulses. For ns pulses, we found a cleaning fluence threshold of $50 \mathrm{~mJ} / \mathrm{cm}^{2}$ that is independent of the pulse durations ( $2.5 \mathrm{~ns}$ and $8 \mathrm{~ns})$ and the wavelengths $(532 \mathrm{~nm}$ and $583 \mathrm{~nm})$ used. The application of ps pulses (FWHM $=30 \mathrm{ps}, \lambda=$ $583 \mathrm{~nm}$ ) lowered this threshold to $20 \mathrm{~mJ} / \mathrm{cm}^{2}$. Both cleaning thresholds are far below the melting thresholds for these laser parameters. Cleaning efficiencies $>90 \%$ were reached for both pulse durations.
\end{abstract}

PACS: $81.65 . C ; 79.60 . \mathrm{Bm}$

The removal of particles from $\mathrm{Si}$ surfaces is a permanent problem for the electronic industry, as such initial contaminants may disturb the subsequent structuring processes. Because of the decreasing size of the structures on microelectronic chips, it is necessary to remove particles with increasingly smaller sizes. Unfortunately, conventional methods such as ultrasonics cannot provide the necessary forces to remove particles with dimensions on the nanometer scale with high efficiencies [1]. Therefore, new techniques have to be developed. One promising idea is the use of short laser pulses for particle removal, the so-called laser cleaning process [2-8].

In the case of the steam laser cleaning process, a thin liquid film is condensed onto the substrate, and subsequently the system is irradiated with a short laser pulse. The energy

*Corresponding author. (E-mail: mario.mosbacher@uni-konstanz.de)

COLA'99 - 5th International Conference on Laser Ablation, July 19-23, 1999 in Göttingen, Germany absorption in the substrate leads to a fast temperature increase both in the substrate and, because of heat transport, also in the liquid film. Bubble nucleation at the solid/liquid interface and the subsequent explosive vaporization of the liquid cause the removal of contaminants.

Here we report on quantitative measurements of the efficiency of steam laser cleaning that uses ns and ps pulses with different wavelengths and pulse durations. For the determination of the efficiency, we use colloidal particles with a well-defined size as model contaminants, deposited as isolated particles on the Si surface.

\section{Experimental details}

\subsection{Sample preparation}

For a systematic investigation of the steam laser cleaning process, it is necessary to use particles with a well-defined geometry whose properties such as size or material can be varied in a controlled way. Since this is not possible with the irregularly shaped alumina particles commonly used, we changed to small monodisperse polystyrene (PS) spheres [9]. For the experiments reported here, we used spheres with a diameter of $800 \mathrm{~nm}$; a different set of experiments $[10,11]$ was carried out with particle diameters ranging from 60 to $800 \mathrm{~nm}$. All these different particles were prepared on the Si wafers in the same way: after rarefaction with isopropyl alcohole (IPA) the particle suspension was deposited on the wafer by a spin coating process. For samples with isolated particles to be obtained, the coagulation due to capillary forces during the drying process had to be kept to a minimum. This was achieved by high evaporation rates of the colloidal suspension and a low concentration of colloid spheres in it. In this way, we were able to prepare samples with mainly isolated spheres (>95\%, cf. Fig. 1) at high particle densities (the mean distance obtained from image processing was about $10 \mu \mathrm{m})$. Details on this procedure will be published elsewhere. 


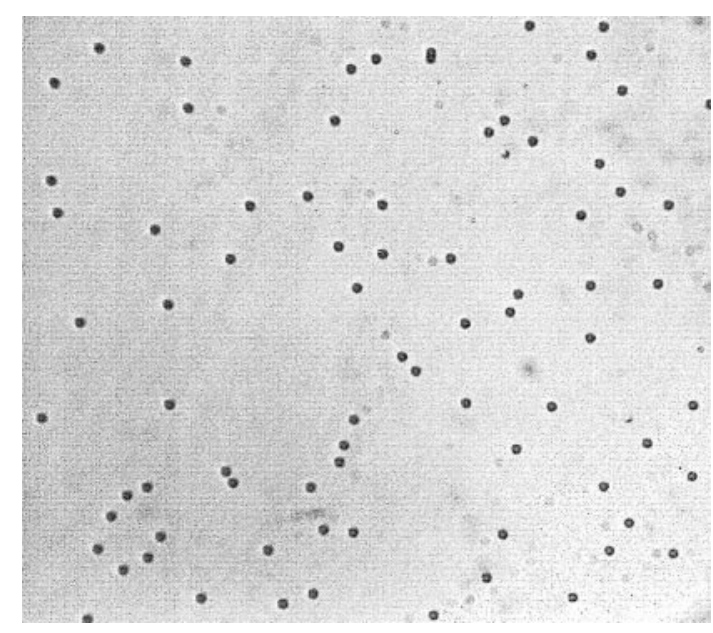

Fig. 1. A typical sample $(4.3 \mu \mathrm{m} \times 5 \mu \mathrm{m})$ before cleaning showing a random distribution of isolated colloidal PS spheres with a diameter of $480 \mathrm{~nm}$

\subsection{Efficiency evaluation}

The efficiency of the process was determined by the measurement of the particle concentration via light scattering. For this purpose, we used a 5-mW HeNe laser focused at the center of the cleaned region. The specular reflection of this probe laser beam was blocked and the scattered light detected by a photodiode. For the improvement of the sensitivity of the light detection system, the HeNe laser beam was chopped at a frequency of about $1 \mathrm{kHz}$ by means of an acousto-optic modulator, and the detector signal was measured with a lockin amplifier phase-locked to the frequency of the chopper. The size of the region illuminated by the probe was on the order of $100 \mu \mathrm{m}$. This is much smaller than the cleaned area; thus it was ensured that a homogeneously irradiated region was monitored. At the same time, this size was sufficiently large to cover several hundred particles at the concentration used, ensuring a small statistical error by monitoring several hundred lift-off processes at once.

Since the prepared samples consist of mainly isolated particles that are not geometrically correlated, the scattered intensity $I$ is essentially directly proportional to the number $N$ of particles in the scattering area, which has been checked in a different set of experiments [11]. For that reason, we will denote the quantity $\left(1-I / I_{0}\right)$ as "cleaning efficiency"; the symbols $I_{0}$ and $I$ denote the scattered intensity before and after the cleaning, respectively.

\subsection{Cleaning setup}

The liquid film for the steam cleaning was obtained by condensation of a burst of water/alcohol (10\% IPA) vapor supplied through a nozzle above the wafer surface just before the arrival of the laser cleaning pulse to the surface [4]. By ellipsometric measurements, we determined the thickness of the liquid layer to be approximately $300 \mathrm{~nm}$.

The cleaning process was carried out using two different pump laser systems providing several different pulse durations and wavelengths: An Nd:YAG laser $(\lambda=532 \mathrm{~nm}$, $\mathrm{FWHM}=8 \mathrm{~ns}$ or $2.5 \mathrm{~ns}$ ) and a pulsed dye amplifier seeded either with a cw dye laser beam or with a synchronously pumped mode-locked dye laser [12] $(\lambda=583 \mathrm{~nm}, \mathrm{FWHM}=$ $2.5 \mathrm{~ns}$ or $30 \mathrm{ps}$ ). The pump laser beam sizes at the sample site were about $1 \mathrm{~mm}^{2}$. Determination of the laser fluence is described in detail in $[13,14]$.

\section{Results}

\section{1 ns pulses}

Results of the energy dependence of the removal efficiency of PS spheres (diameter $800 \mathrm{~nm}$ ) by the steam cleaning process with the application of ns pulses (FWHM $=8 \mathrm{~ns}, \lambda=$ $532 \mathrm{~nm} ; \mathrm{FWHM}=2.5 \mathrm{~ns}, \lambda=532 \mathrm{~nm} ; \quad F W H M=2.5 \mathrm{~ns}$, $\lambda=583 \mathrm{~nm}$ are plotted in Fig. 2. As has already been shown in a previous examination [10], the removal process for our samples is completely statistical, making quantitative studies feasible. Thus we included in the figure only the results obtained for a cleaning process induced by 20 consecutive shots at a fixed fluence for better illustration.

In another work [11], we were able to show that for steam laser cleaning with laser pulses of FWHM $=8 \mathrm{~ns}$ and $\lambda=532 \mathrm{~nm}$, there exists a universal cleaning threshold of about $50 \mathrm{~mJ} / \mathrm{cm}^{2}$ for the removal of particles with diameters of $60-800 \mathrm{~nm}$. This threshold value is also found in the experiments presented here. An explanation of this identical threshold value for all three sets of laser parameters can be given from the underlying physical process responsible for the cleaning effect - the nucleation and growth of bubbles within the heated liquid. This nucleation is a direct consequence of the heat transfer from the substrate to the liquid, and thus the dynamics of bubble growth is governed by the transfer of heat into the liquid layer. Especially the penetration of the $373 \mathrm{~K}$ isotherm into the liquid layer limits the bubble growth velocity, as bubble growth in layers with temperatures below $373 \mathrm{~K}$ is impossible.

Numerical simulations [15] were applied to compute temperature profiles of the liquid layer for the pulse parameters

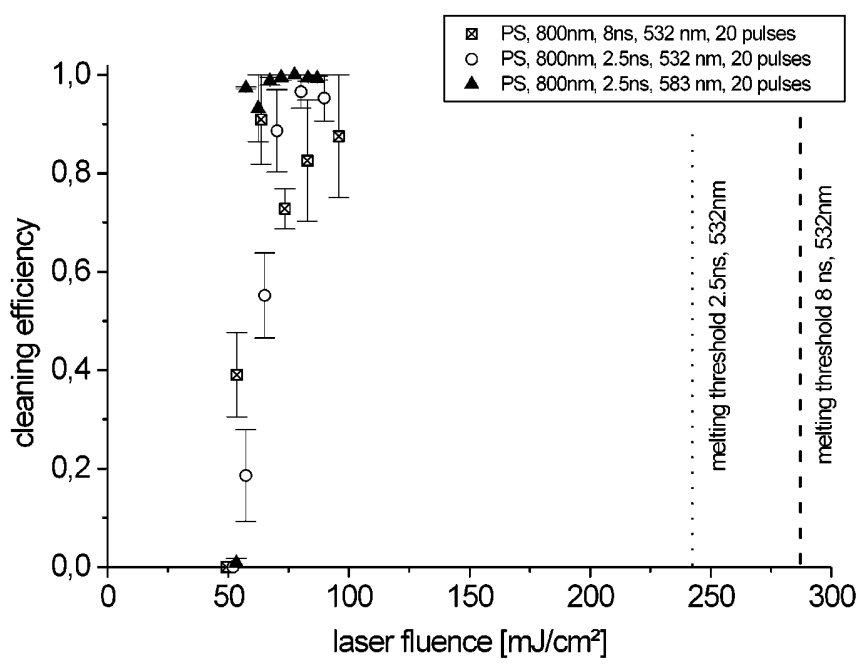

Fig. 2. Cleaning efficiency for steam laser cleaning of $800 \mathrm{~nm}$ PS spheres using ns pulses of several durations at different wavelengths. The results correspond to a cleaning process induced by 20 consecutive laser shots at a fixed fluence 
used. A comparison of the results shows similar spatial temperature profiles for the layers adjacent to the Si surface by the time the surface reaches its maximum peak temperature, especially for the layer of liquid heated to more than $373 \mathrm{~K}$. From there, one should expect the same cleaning thresholds (determined by the onset of bubble nucleation) for the different laser parameters applied in our experiments. It should be noted, however, that as soon as the wavelength decreases to the UV, there should be a different threshold, because of the small optical penetration depth in $\mathrm{Si}$.

Above the cleaning threshold, we again obtain a similar behavior for all three sets of laser parameters. After a steep increase just above the threshold, cleaning efficiencies of $>90 \%$ (after 20 cleaning steps) are reached at about $75 \mathrm{~mJ} / \mathrm{cm}^{2}$ for the 2.5 -ns pulses and at about $100 \mathrm{~mJ} / \mathrm{cm}^{2}$ for the 8-ns pulse. We point out that these high cleaning efficiencies are reached at energy densities that are lower by a factor of 2-3 than the melting thresholds of a bare Si surface for the given laser parameters. With respect to industrial applications where surface damage has to be avoided, this result is one of the major advantages of the steam laser cleaning process as compared with the dry laser cleaning. In dry laser cleaning, in which the thermal expansion of the substrate is responsible for the particle removal, high cleaning efficiencies with ns pulses are reached only at energy densities much closer to the melting threshold [16].

\section{2 ps pulses}

When the pulse duration is decreased from ns to ps, the amount of heat energy diffusing in depth in the Si wafer during the pulse absorption lowers. In particular, the thermal diffusion lengths in Si at $583 \mathrm{~nm}$ for an 8-ns and a 30-ps laser pulse are $1.26 \mu \mathrm{m}$ and $109 \mathrm{~nm}$, respectively. The comparison of these lengths to the optical penetration depth in $\mathrm{Si}$ at $583 \mathrm{~nm}, 1.7 \mu \mathrm{m}$, indicates that for the ps pulses, the fluence required to bring the surface to a given peak temperature is smaller than for ns pulses.

In view of the investigation of the physical processes of steam laser cleaning, when one changes from ns to ps pulses there are two important consequences. First, the melting threshold of the bare $\mathrm{Si}$ surface will decrease somewhat. Second, the shorter pulse length will affect the cleaning threshold. In addition to the heat conduction inside the wafer on this threshold shift, another process becomes important: the nucleation of bubbles. High cleaning efficiencies are reached only by the heating of the substrate/liquid interface as well as a sufficiently thick liquid layer to allow bubble nucleation and growth. When applying ps pulses, several scenarios for the latter might be possible. Due to the shorter pulse length, a higher superheating of the liquid can be achieved, which leads to higher bubble growth velocities and therefore to higher cleaning efficiencies. Another possibility would be that the heating of the liquid layer takes place on a time scale longer than the ps pulse duration and the heating of the Si. This would lead only to a small reduction in the cleaning threshold.

Because of the existence of effects affecting the steam cleaning process in opposite directions when the pulse duration is reduced, we experimentally investigated the influence of pulse duration on the cleaning threshold and the behavior of the cleaning efficiency.
For a comparison between ns and ps steam laser cleaning, we plotted in Fig. 3 the cleaning efficiency as a function of laser fluence for pulses with FWHM $=30 \mathrm{ps}$ and $\lambda=583 \mathrm{~nm}$, as well as for ns pulses with FWHM $=2.5 \mathrm{~ns}$ and $\lambda=532 \mathrm{~nm}$. Since we found the same cleaning threshold for" all ns, the experiments have included only those obtained at $532 \mathrm{~nm}, 2.5 \mathrm{~ns}$. For the ps laser system, the energy output fluctuations are much higher pulse by pulse than those for the ns systems. These fluctuations were monitored and result in the horizontal error bars in Fig. 3. The influence of the energy fluctuations in the ns regime was negligible $(\leq 5 \%)$ and thus is not included as error bars.

As for ns pulses, we found a sharp cleaning threshold for the ps steam laser cleaning; it is, however, much lower: about $20 \mathrm{~mJ} / \mathrm{cm}^{2}(\mathrm{FWHM}=30 \mathrm{ps}, \lambda=583 \mathrm{~nm})$. The numerical simulations reveal for this fluence similar peak surface temperatures of the $\mathrm{Si}$ as for the ns cleaning threshold. This decrease in the threshold value is expected from the scenario of energy absorption and heat diffusion in the substrate described above.

Of importance with respect to applications is also the regime where efficient cleaning takes place, i.e, the fluence interval where cleaning efficiency exceeds, e.g., $90 \%$. This is found at fluences of $>750 \mathrm{~mJ} / \mathrm{cm}^{2}$ for both pulse durations.

For semiconductors, the melting threshold can be determined experimentally by means of real-time reflectivity measurements with ps resolution [17], because of the high reflectivity of the liquid phase. The threshold value for the onset of melting of the clean Si wafer was found to be approximately $220 \mathrm{~mJ} / \mathrm{cm}^{2}$; this is in good agreement with our numerical simulations and is the value already determined for $\mathrm{FWHM}=20 \mathrm{ps}, \lambda=532 \mathrm{~nm}$ [18].

Efficient cleaning takes place at energy densities below the melting threshold by a factor of 3 , as is the case for ns pulses. This is sufficient for safe application of the process. Comparing the ratios of melting threshold to cleaning threshold, we obtained a value of 5 for the ns pulses but a factor of 11 for the ps regime.

The decrease of the cleaning threshold and the fact that with the application of ps pulses, efficient particle removal can be achieved, may indicate that still the time scale of substrate heating is not too short to allow enough heat diffusion

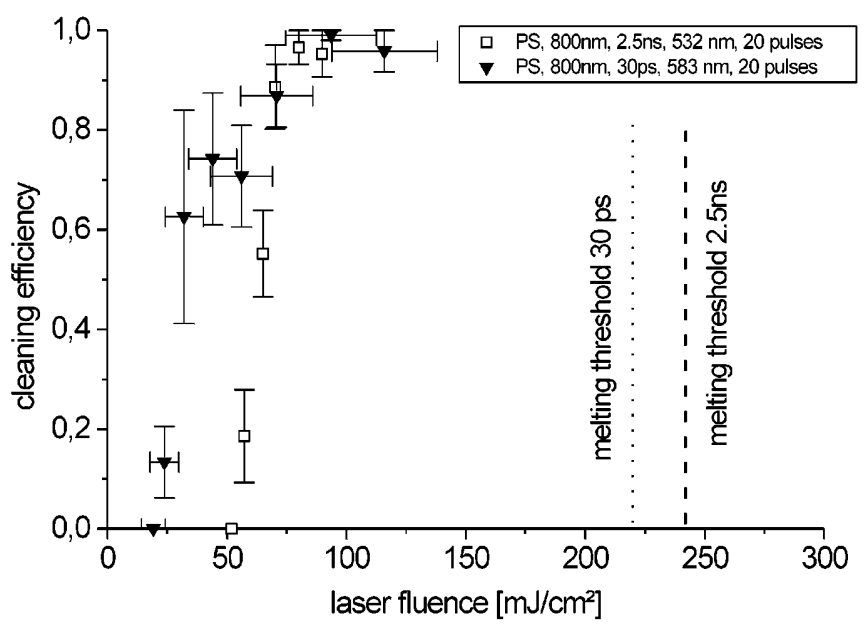

Fig. 3. Comparison of the cleaning efficiencies of ns and ps steam laser cleaning $(\mathrm{FWHM}=2.5 \mathrm{~ns}$ and $30 \mathrm{ps})$ 
into the liquid. On the other hand, the ratio of melting threshold to efficient cleaning threshold remained nearly constant in our experiments. This could be taken as support for the assumption that much shorter pulses would not result in an increase in efficiency of the process. However, in order to further investigate this and to reveal the role of superheating, time-resolved experiments on bubble nucleation upon ps-pulse laser irradiation must be performed.

\section{Conclusion}

We have demonstrated the feasibility of steam laser cleaning with ps pulses for the first time and have been able to quantitatively compare the cleaning efficiency to that achieved with ns pulse experiments. The latter reveals a cleaning threshold of $50 \mathrm{~mJ} / \mathrm{cm}^{2}$, and no significant influence of pulse duration ( 2.5 and $8 \mathrm{~ns}$ ) or wavelength (532 and $583 \mathrm{~nm}$ ) was found. For ps cleaning pulses, we obtained a cleaning threshold of $20 \mathrm{~mJ} / \mathrm{cm}^{2}(30 \mathrm{ps}, 583 \mathrm{~nm})$. Cleaning efficiencies of more than $90 \%$ are reached for both ns and ps pulses at fluences about a factor of 3 lower than the melting threshold of the substrate, which has been determined to be $220 \mathrm{~mJ} / \mathrm{cm}^{2}$ for the case of ps pulses. Thus for industrial applications concerning the suppression of substrate damage, both ns and ps steam laser cleaning seem to be promising when compared to traditional methods.

Acknowledgements. This work was supported by the Optikzentrum Konstanz and the EU (TMR ERB-CT98-0188, "Modeling and diagnostic of pulsed laser-solid interactions: applications to laser cleaning"). N. Chaoui acknowledges the funding of his stay at the Instituto de Optica, CSIC (Madrid) through the TMR ERB-CT98-0188. J. Siegel acknowledges with pleasure the funding of the European Union through a Marie Curie Fellowship (ERB0001GT954352) within the TMR program. We thank the group of J.V. Garcia-Ramos at the Instituto de Estructura de la Materia, CSIC, Madrid for generous support in experimental needs. Wacker Siltronic supplied the industrial silicon wafers.

\section{References}

1. J. Bardinan: In Particles on surfaces, ed. by K.L. Mittal (Plenum, New York 1988) p. 329

2. W. Zapka, W. Ziemlich: Appl. Phys. Lett. 58(20), 2217 (1991)

3. K. Imen, J. Lee, S.D. Allen: Appl. Phys. Lett. 58(2), 203 (1991)

4. A.C. Tam, W.P. Leung, W. Zapka, W. Ziemlich: J. Appl. Phys. 71(7), 3515 (1992)

5. J.B. Heroux, S. Boughaba, I. Ressejac, E. Sacher, M. Meunier: J. Appl. Phys. 79, 2857 (1996)

6. Y.F. Lu, W.D. Song, K.D. Ye, M.H. Hong, D.M. Liu, D.S.H. Chan, T.S. Low: Appl. Surf. Sci. 120, 317 (1997)

7. A.C. Engelsburg: Proc. SPIE 3274, 100 (1998)

8. G. Vereecke, E. Röhr, M.M. Heyns: J. Appl. Phys. 85(7), 3837 (1999)

9. Interfacial Dynamics Corporation, Portland, Or 97224 USA

10. P. Leiderer, J. Boneberg, M. Mosbacher, A. Schilling, O. Yavas: Proc. SPIE 3274, 68 (1998)

11. M. Mosbacher, V. Dobler, J. Boneberg, P. Leiderer: (unpublished)

12. J. Siegel, J. Solis, C.N. Afonso, C. Garcia: J. Appl. Phys. 80, 6677 (1996)

13. J. Siegel: In Structural transformation dynamics in Ge films upon ultrashort laser pulse irradiation (Ph.D. thesis, Universidad Autonoma de Madrid 1999)

14. J. Solis, C.N. Afonso, S.C.W. Hyde, N.P. Barry, P.M.W. French: Phys. Rev. Lett. 76(14), 2519 (1999)

15. J. Bischof: In Metallische Dünnfilmschmelzen nach Pulslaserbestrahlung: Phasenumwandlungen und Instabilitäten (Ph.D. thesis, University of Konstanz 1996)

16. M. Mosbacher, V. Dobler, J. Boneberg, P. Leiderer: CLEO/Europe EQEC (1998), CPD2.12 (1998)

17. J. Siegel, J. Solis, C.N. Afonso: Appl. Phys. Lett. 75, 1071 (1999)

18. L.A. Lompre, J.M. Liu, H. Kurz, N. Bloembergen: Appl. Phys. Lett. 430, 168 (1983) 